\title{
Iniquidades sociais no consumo alimentar no Brasil: uma revisão crítica dos inquéritos nacionais
}

\author{
Social inequities in food consumption in Brazil: \\ a critical review of the national surveys
}

Raquel Canuto (https://orcid.org/0000-0002-4042-1913) ${ }^{1}$

Marcos Fanton (https://orcid.org/0000-0001-5360-3647) ${ }^{2}$

Pedro Israel Cabral de Lira (https://orcid.org/0000-0002-1534-1620) ${ }^{3}$

\footnotetext{
1 Programa de

Pós-Graduação em

Alimentos, Nutrição e

Saúde,Universidade Federal

do Rio Grande do Sul. R.

Ramiro Barcelos 2400, Rio

Branco. 90035-003 Porto

Alegre RS Brasil.

raquel.canuto@ufrgs.br

${ }^{2}$ Programa de Pós-

Graduação em Filosofia,

Universidade Federal de

Santa Maria. Santa Maria

RS Brasil.

${ }^{3}$ Programa de Pós-

Graduação em Nutrição,

Universidade Federal de

Pernambuco. Recife PE

Brasil.
}

\begin{abstract}
The scope of this article is to investigate the association between socioeconomic status and food consumption among Brazilians and understand how the results of the major national surveys contribute to the identification of possible social and ecological inequities in food consumption. A systematic review of studies conducted a search for publications that analyzed the relationship between socioeconomic position and food consumption in major national surveys. The definition of socioeconomic position, the selection of its indicator variables and the critical analysis of its operationalization were based on ecosocial epidemiology. Individuals with lower socioeconomic status (skin color/brown or black-skinned, less education and income, and being resident in rural areas) are less likely to have a diversified and healthy diet. The effects of gender and marital status can only be understood by social profiling. Socioeconomic position variables create a complex web of causality that helps to explain the reasons that determine the food consumption of Brazilians and reveal social inequities in diet and nutrition. Greater conceptual clarity regarding the measurement of socioeconomic parameters is required to achieve greater precision regarding the causes of social inequalities in food.

Key words Food consumption, Health inequities, Social inequalities, Ecosocial epidemiology
\end{abstract}

Resumo O objetivo deste artigo é investigar a associação entre posição socioeconômica e o consumo alimentar entre os brasileiros para poder compreender como os grandes inquéritos nacionais estão contribuindo na identificação de desigualdades sociais e ecológicas neste último. Revisão sistemática da literatura de publicações que analisaram a relação entre posição socioeconômica e consumo alimentar nos grandes inquéritos nacionais. A definição de posição socioeconômica, seleção das suas variáveis indicadoras e a análise crítica de sua operacionalização basearam-se na epidemiologia ecossocial. Indivíduos em menor posição socioeconômica - cor da pele/raça parda ou negra, menor escolaridade e renda e de áreas rurais - têm menor probabilidade de consumir uma alimentação diversificada e saudável. Já os efeitos do gênero e da situação conjugal só poderiam ser melhor compreendidos com um recorte social. As variáveis de posição socioeconômica formam uma complexa teia de causalidade que ajuda a explicar a determinação do consumo alimentar dos brasileiros e a denunciar as iniquidades sociais na alimentação e nutrição. Há a necessidade de maior clareza conceitual a respeito da medição dos parâmetros para alcançar maior precisão sobre as causas das desigualdades sociais em alimentação.

Palavras-chave Consumo alimentar, Iniquidades em saúde, Desigualdades sociais, Epidemiologia ecossocial 


\section{Introdução}

As doenças crônicas não transmissíveis (DCNT) são a principal causa de mortalidade no Brasil ${ }^{1}$. Em parte, isso é explicado pela rápida transição nutricional experienciada pelo país nas últimas décadas. $O$ processo de urbanização e industrialização, consequentes do desenvolvimento econômico brasileiro, somados a globalização, mudaram a forma como se produz, distribui e consome os alimentos. A partir disso, pode-se observar as altas prevalências de desnutrição serem gradualmente substituídas pelo sobrepeso e obesidade $^{2}$.

Contudo, a distribuição desses agravos não ocorre de forma homogênea na população e pode revelar importantes iniquidades sociais. Já está bem estabelecida na literatura a influência das características socioeconômicas nos hábitos de vida, nos agravos crônicos e na expectativa de vida ${ }^{3}$. A direção da associação entre nível socioeconômico e obesidade, por exemplo, varia de acordo com o nível de desenvolvimento dos países. Nos países desenvolvidos, os indivíduos com menor escolaridade e renda são mais propensos a desenvolver obesidade do que indivídeuos de grupos socioeconômicos mais elevados. No entanto, nos países da baixa e média renda, essa associação é inversa ${ }^{4,5}$. Já a associação entre nível socioeconômico e qualidade da dieta - um importante mediador na relação entre nível socioeconômico e obesidade -, embora esteja bem estabelecida em países desenvolvidos ${ }^{6}$, ainda é pouco estudada em países de baixa e média renda, como o Brasil'.

A fim de qualificar as medidas dos estudos epidemiológicos nas investigações sobre iniquidades sociais em saúde, Nancy Krieger elaborou uma teoria epidemológica sensível aos contextos social e ecológico, às trajetórias de vidas dos indivíduos e conflitos intergeracionais e às interrelações entre pessoas de diferente nível social e econômico. Esta teoria é denominada por Krieger de "teoria ecossocial da distribuição da doença" e tem, como tese central, a incorporação biológica de exposições decorrentes do contexto social e ecológico e de relações históricas entre classes sociais e econômicas.

A partir disso, em 1997, Krieger et al. ${ }^{8}$ propuseram as categorias de classe social e de posição socioeconômica. Tratam-se de conceitos complexos e multidimensionais, nos quais os indivíduos são classificados comparativamente a outros indivíduos em grupos interdependentes. Para existirem, classes sociais dependem do modo como as instituições da sociedade, em termos econômicos e jurídico, são estruturadas. Uma classe social é sempre interdependente e definida de acordo com seu correlato (como empregado e empregador, homem e mulher). Posição socioeconômica, por outro lado, é um conceito ainda mais específico. É definido de acordo com a expressão, em dada sociedade, das distribuições de diferentes atributos materiais e não materiais, como medidas baseadas em recursos (bens materiais e sociais) e medidas baseadas no prestígio (status do indivíduo numa hierarquia social). Dessa forma, além das medidas tradicionais de nível socioeconômico, estão incluídas variáveis como características de gênero e orientação sexual, privação social e material, raça e etnia e variáveis da situação conjugal e da posição de trabalho. Os autores ${ }^{8}$ também destacam a importância de medidas em nível individual, familiar e de vizinhança, defendendo que a posição socioeconômica, em parte, determina o acesso individual a bens e serviços, como alimentação, atividade física e outras práticas saudáveis, que estão associados diretamente à ocorrência das DCNT ${ }^{9,10}$.

O Brasil é um país extramente desigual e foi o primeiro país a criar a sua própria comissão nacional sobre determinantes sociais da saúde (CNDSS), em 2006. Com isso, integrou-se ao movimento global em torno do assunto e deu um passo fundamental para a discussão da importância dos determinantes sociais na situação de saúde dos brasileiros e da necessidade de enfrentar as iniquidades em saúde geradas por estes ${ }^{10}$. Porém, mesmo nos materiais publicados pela CNDSS, não são discutidos de modo mais amplo os determinantes sociais da alimentação e nutrição.

Nesse contexto, por meio da revisão de estudos que analisaram os dados dos grandes inquéritos populacionais brasileiros, investigou-se a associação entre posição socioeconômica e consumo alimentar. Além disso, a partir da teoria epidemiológica proposta por Nancy Krieger, analisou-se como os resultados das pesquisas nacionais estão contribuindo para que sejam identificadas possíveis iniquidades no consumo alimentar da população brasileira influenciadas por iniquidades sociais e ecológicas.

\section{Metodologia}

Uma revisão sistemática da literatura foi conduzida com o objetivo de recuperar artigos originais que tenham investigado a relação entre po- 
sição socioeconômica e consumo e/ou aquisição de alimentos nas pesquisas nacionais. A definição de posição socioeconômica e a seleção das variáveis indicadoras de posição socioeconômica foram baseadas no referencial teórico proposto por Krieger, ainda que nem todas variáveis propostas sejam incluídas ${ }^{8}$. Dessa forma, os artigos recuperados da literatura cumpriam com os seguintes critérios de inclusão: (1) ter como desfecho hábitos alimentares, disponibilidade ou consumo alimentar medido de forma quantitativa ou qualitativa; (2) ter como exposição indicadores de posição socioeconômica, como sexo, educação, renda, cor da pele/raça, situação conjugal, área de residência ou outras variáveis socioambientais; (3) incluírem a população de adultos; (4) serem publicados na forma de artigos ou relatórios de pesquisa que tenham utilizado dados das pesquisas com amostragem com representatividade nacional [Pesquisa de Orçamentos Familiares (POF), Inquérito Nacional de Alimentação (INA), Sistema de Vigilância de Fatores de Risco e Proteção para Doenças Crônicas por Inquérito Telefônico (Vigitel) e a Pesquisa Nacional de Saúde (PNS)].

Abaixo, segue uma rápida descrição de cada uma das pesquisas. As POF ocorrem desde 1987, no Brasil e, entre os anos 2002-03 e 2008-9, disponibilizam informações transversais sobre o tipo e a quantidade de alimentos adquiridos nos domicílios brasileiros, por setes dias consecutivos, a partir de uma amostra probabilística com representatividade dos meios urbanos de todas as unidades da federação e para o meio rural das cinco grandes regiões brasileiras ${ }^{11}$. Já o INA é a primeira pesquisa sobre o consumo alimentar individual do Brasil com uma subamostra da POF (2008-9) com a mesma representatividade. No INA, o consumo alimentar foi avaliado por meio de um registro alimentar de dois dias não consecutivos em indivíduos com 10 anos ou mais ${ }^{12}$. O Vigitel, por sua vez, é um inquérito telefônico transversal que pretende monitorar os principais fatores de risco e proteção das DCNT, representativo apenas para a população adulta (idade $\geq 18$ anos) residente nas 26 capitais do Brasil e no Distrito Federal, realizado anual e continuamente desde $2006^{13}$. Por fim, a PNS (2013) averiguou o conjunto de indicadores de consumo alimentar compatível aos do Vigitel por entrevistas domiciliares. Trata-se de um inquérito transversal representativo de toda população adulta (idade $\geq 18$ anos) do país (rural e urbana) ${ }^{14}$.

As buscas pelos artigos foram realizadas nas bases do PubMed e Scielo, além da busca por do- cumentos governamentais em dezembro de 2016 (uma atualização foi feita em julho de 2017). Foram utilizados termos de busca relativos aos critérios de inclusão acima descritos (termos utilizados no Pubmed encontram-se na Quadro 1). A seleção dos relatórios se deu de forma ativa nos sítios do Instituto Brasileiro de Geografia e Estatística (IBGE) e do Ministério da Saúde.

$\mathrm{Na}$ seleção dos artigos, em um primeiro momento, foram lidos todos os títulos e resumos. No segundo momento, os manuscritos foram lidos na íntegra e a sua inclusão no estudo dependeu do atendimento aos critérios estabelecidos. Artigos adicionais foram identificados a partir das listas de referência dos artigos selecionados. Já os relatórios foram lidos na íntegra, extraindose as informações que não constavam nos artigos selecionados. O software ENDNOTE X foi utilizado no processo de armazenamento e seleção dos artigos.

Quadro 1. Buscas no PubMed.

\begin{tabular}{|c|c|}
\hline Exposição & 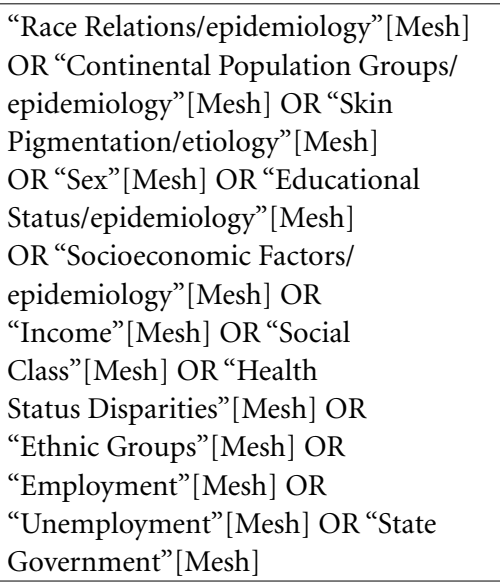 \\
\hline Desfecho & 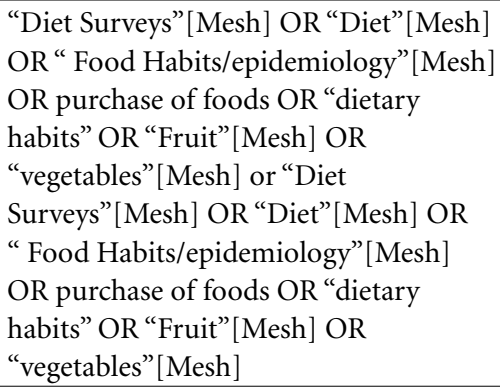 \\
\hline Estudo & $\begin{array}{l}\text { "Brazil"[Mesh] AND "Brazilian Family } \\
\text { Budgets Survey" OR "National Dietary } \\
\text { Survey" OR "National Health Survey" } \\
\text { OR "surveillance system of risk factors } \\
\text { for chronic non-communicable } \\
\text { diseases" }\end{array}$ \\
\hline Limites & Humans; Adult: $19-44$ years \\
\hline
\end{tabular}


Os dados dos documentos (artigos e relatórios) foram extraídos, sumarizados e apresentados em Quadros de acordo com as seguintes informações: primeiro autor, ano de publicação, nome do estudo, ano da coleta de dados, faixa etária dos participantes, variáveis de posição socieconômica investigadas, desfechos investigados e resultados das associações entre as variáveis de posição socioeconômica e acesso ou consumo alimentar. O conjunto de resultados foi analisado de forma qualititativa.

Após a sumarização dos resultados, a partir do referencial teórico adotado pelo estudo (epidemiologia ecossocial), foi realizada uma análise crítica das variáveis de posição socioeconômicas incluídas nas publicações e/ou inquéritos. Além disso, foram feitas proposições sobre os seu uso em futuros estudos que visem denunciar iniquidades sociais em saúde no Brasil.

\section{Resultados}

As buscas resultaram em 689 artigos recuperados $($ Pubmed $=541$, Scielo $=148)$. Foram lidos todos os títulos e abstracts, cuja seleção resultou em 27 artigos para leitura na íntegra. Com a exclusão de artigos sobrepostos após a leitura, restaram 24 artigos. Desses, seis apresentavam dados da POF, quatro do INA, 10 do Vigitel e três da PNS. Adicionalmente, foram incluídos dados de três relatórios do Vigitel que traziam informações não apresentadas nos artigos.

$\mathrm{Na}$ Quadro 2, observam-se os dez artigos recuperados que utilizaram dados das POFs e do INA. Três artigos e um relatório apresentam dados da pesquisa de $2002-3^{11,15-17}$ e seis das pesquisas de 2008-9 (POF e INA) ${ }^{18-23}$.

Os seguintes desfechos foram investigados nos estudos: participação relativa de 15 grupos de alimentos estabelecidos ${ }^{11,18}$, consumo de frutas e verduras ${ }^{16}$, consumo de bebidas ${ }^{21}$, alimentos com maior frequência de consumo ${ }^{23}$, disponibilidade domiciliar de calorias ${ }^{15}$, consumo de macronutrientes ${ }^{11,15,18,20,22}$, consumo de micronutrientes $^{20,22}$, padrões alimentares ${ }^{17}$ e classificação dos alimentos consumidos de acordo com o seu nível de processamento industrial ${ }^{19}$ (Quadro 2).

Com relação às variáveis de posição socioeconômica investigadas, o sexo foi incluído nas quatro análises com dados do INA ${ }^{20-23}$. Quando comparados à mulheres, os homens apresentavam maior ingestão de bebidas alcoólicas, arroz, feijão, carne bovina, refrigerantes, batata e salga- dos fritos. Já as mulheres tiveram um maior consumo de café, pão de sal, sucos, refrescos e outras bebidas açucaradas ou adoçadas artificialmente, bolos, óleos e gorduras, sopas e doces, com maior inadequação no consumo de micronutrientes: cálcio, fósforo, ferro, cobre, zinco, selênio, tiamina, piridoxina, vitamina B12. Todos artigos incluíram a renda e esta mostrou-se diretamente associada a uma alimentação mais variada, que incluiu farináceos, carnes, lácteos, frutas, verduras, legumes, gorduras animal e vegetal, além de refrigerantes e bebidas de baixa caloria, bebidas alcoólicas, condimentos e refeições prontas. Os indivíduos de maior renda apresentaram um consumo mais elevado de energia, lipídeos e micronutrientes, exceto o sódio. Quando empregada a análise de componentes principais na determinação dos padrões alimentares, a mesma tendência foi observada: a renda esteve associada diretamente ao consumo do padrão alimentar "duplo". O consumo de alimentos processados e ultraprocessados, apesar de ter crescido ao longo do tempo em todos os estratos, também foi maior nos indivíduos com maior renda. Por outro lado, o maior nível de renda mostrou-se inversamente associado ao consumo de alimentos básicos e tradicionais dos brasileiros, como feijões e outras leguminosas, raízes, tubérculos e seus derivados e açúcar. Indivíduos com menor renda apresentaram maior consumo de carboidratos e fibras alimentares e, por isso, o padrão alimentar tradicional também esteve associado a esse grupo de indivíduos. Apenas três estudos avaliaram o consumo/disponibilidade de alimentos de acordo com a escolaridade ${ }^{17,20,21}$, apresentando resultados semelhantes aos verificados com a renda. Por fim, cinco estudos investigaram as diferenças no consumo de acordo com a área urbana e ru$\mathrm{ral}^{11,17,18,21,22}$. Os moradores da área urbana apresentaram uma tendência de consumo alimentar parecido com os indivíduos de maior renda, ou seja, uma dieta mais variada com predomínio de biscoitos, carnes, lácteos, frutas, legumes, verduras, condimentos e refeições prontas. O consumo de feijões e outras leguminosas, raízes, tubérculos e derivados, ovos, peixes, gordura animal e açúcar foi maior na área rural, apresentando também maiores inadequações no consumo de micronutrientes. A disponibilidade de lipídeos e energia foi maior na área urbana; de carboidratos e proteínas, na área rural. $\mathrm{O}$ consumo de bebidas doces e adoçadas artificialmente foi maior na área urbana, com exceção do café com açúcar, que foi maior na área rural (Quadro 2). 


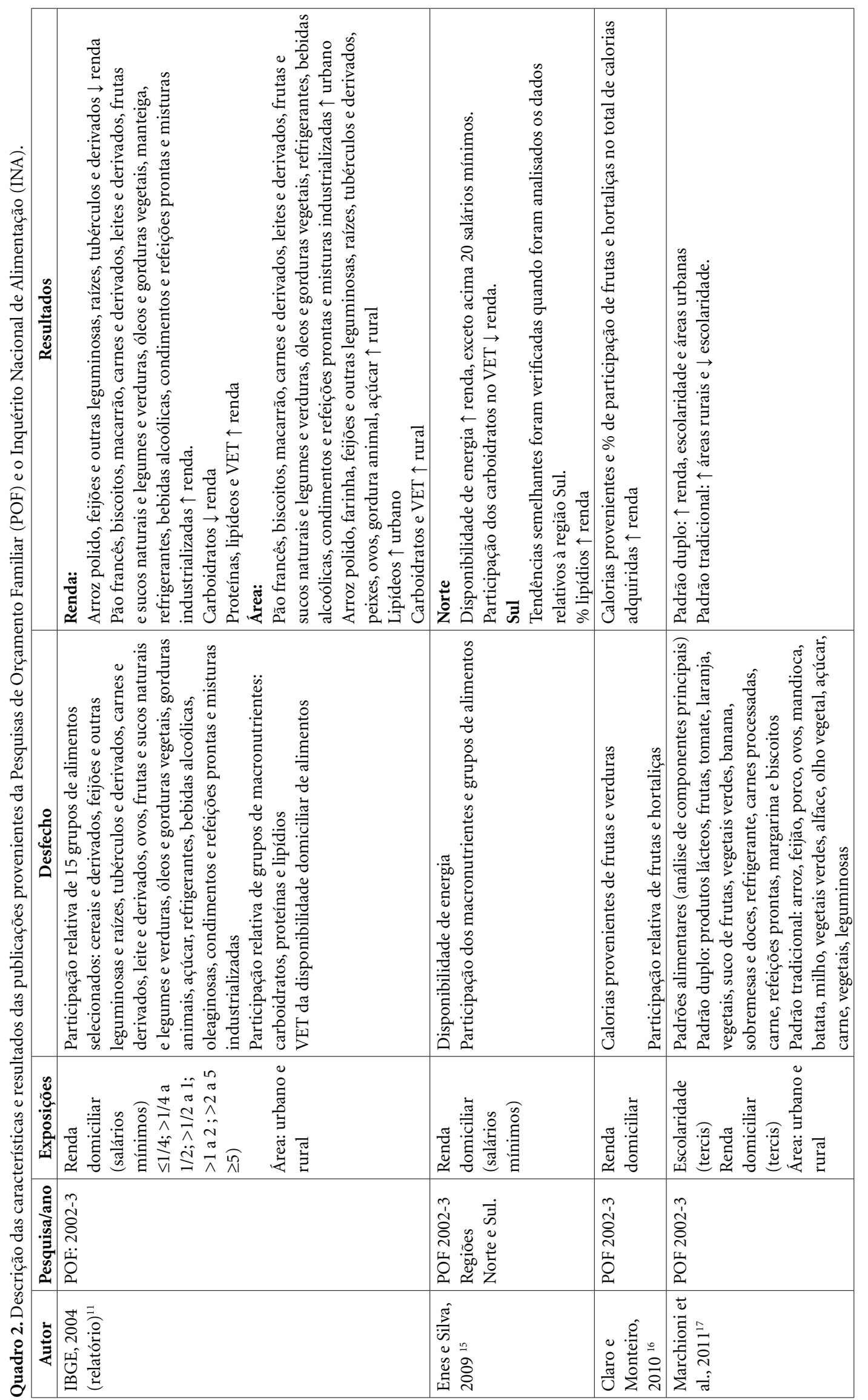




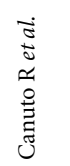

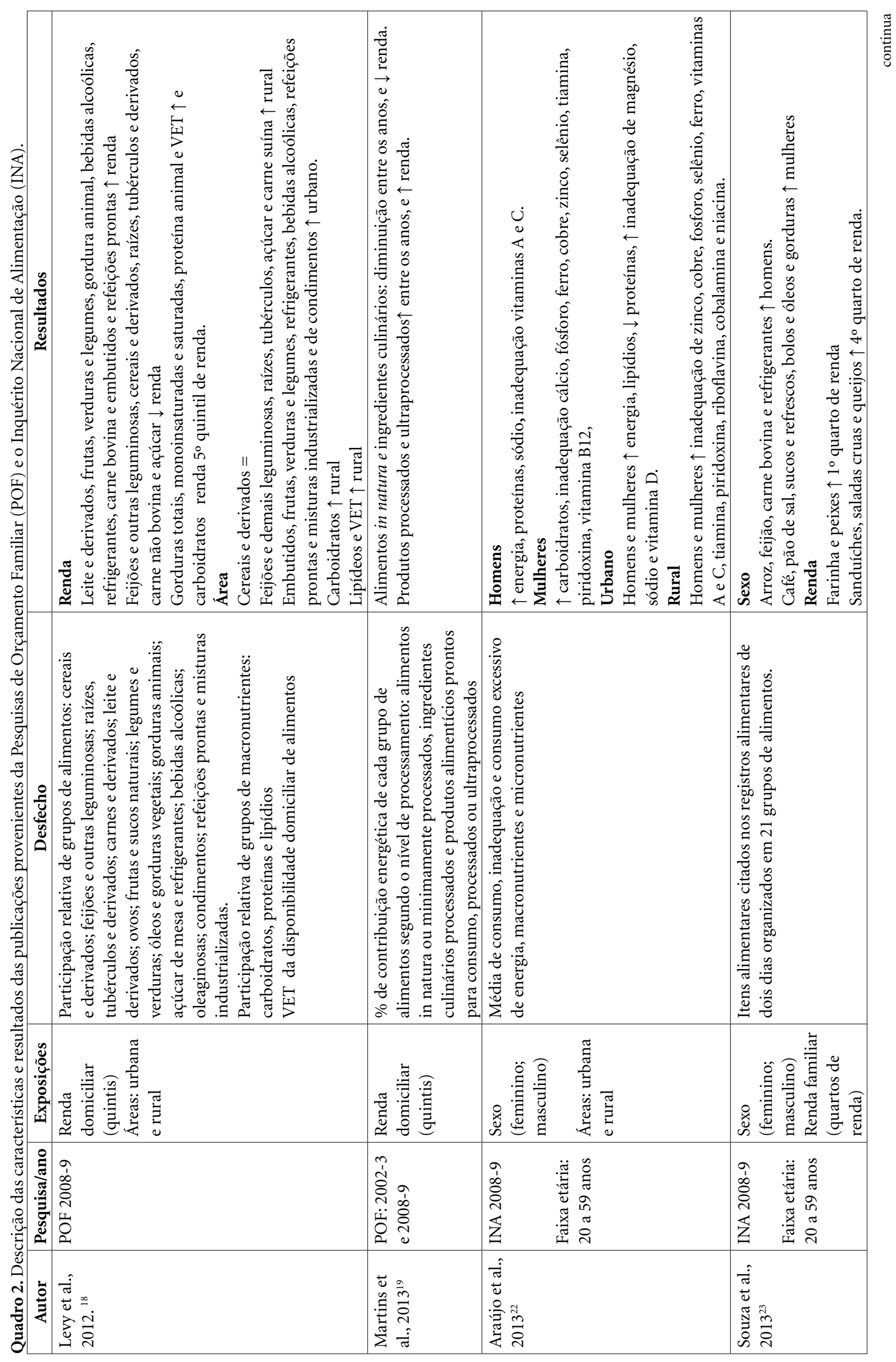




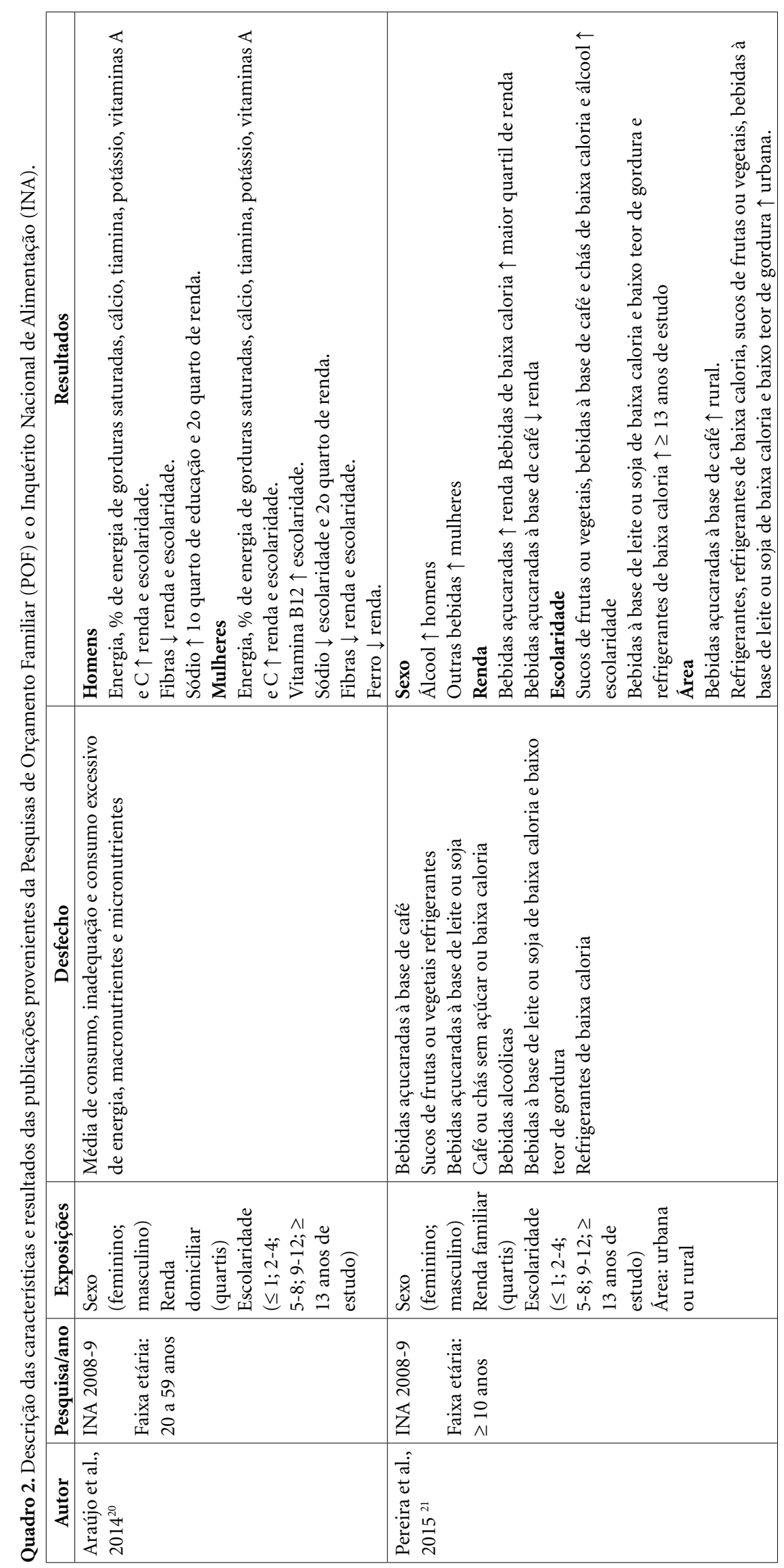


$\mathrm{Na}$ Quadro 3, pode-se observar os resultados dos estudos que tiveram como base dados do Vigitel, dos anos de 2006 a 2016 ${ }^{13,24-35}$, e da PNS de $2013^{35-37}$. Ambos inquéritos investigaram comportamentos de consumo alimentar de risco e proteção para as DCNT de acordo com sexo (todos os estudos), cor da pele/raça ${ }^{26,33,37-39}$, situação conjugal $^{37}$ e escolaridade ${ }^{24-26,32,34-39}$. Analisando os resultados de todas as pesquisas de forma conjunta, independentemente do ano da pesquisa, pode-se observar que o sexo masculino esteve associado ao maior consumo de feijão, carnes com excesso de gordura, leite com teor de gordura, refrigerantes e/ou sucos artificias. Por outro lado, o consumo regular e/ou recomendado de frutas, legumes e verduras e doces foi maior entre as mulheres. A cor da pele/raça preta ou parda esteve associado ao maior consumo de feijão, carnes com excesso de gordura, leite com teor de gordura; já o consumo de doces foi menor entre os pardos e o consumo regular e/ou recomendado de frutas, legumes e verduras foi maior entre os brancos. O estudo que incluiu situação conjugal nas análises demonstrou que ser casado aumenta o consumo de frutas, legumes e verduras ${ }^{24}$. Ressalta-se, por fim, que, em todos os grupos, o consumo de frutas, legumes e verduras está aquém do recomendado.

Alguns marcadores mostram relações mais complexas. O consumo de carnes com excesso de gordura e leite com teor de gordura não apresenta clara associação ao longo dos estudos. O consumo regular de refrigerantes e/ou sucos artificias, nos estudos mais recentes, esteve associado diretamente a homens com escolaridade entre 9 e 11 anos de estudo $e$, inversamente, às mulheres com escolaridade $\geq 12$ anos de estudo. Pertencer à área urbana foi associado ao maior consumo de frutas, legumes e verduras, refrigerantes e/ou sucos artificias e doces. Já pertencer à área rural foi associado ao maior consumo de carnes com excesso de gordura. A partir de 2013, o Vigitel incluiu a investigação do consumo de lanches no lugar nas principais refeições, que se mostrou associado às mulheres e a indivíduos com maior escolaridade.

\section{Discussão}

Esta revisão da literatura foi a primeira a traçar a influência da posição socioeconômica no acesso/ consumo de alimentos dos brasileiros, demonstrando como gênero, cor da pele/raça, situação conjugal, escolaridade, renda e área de moradia podem estar associados ao consumo dos alimen- tos no Brasil. Além disso, possibilitou identificar como as publicações decorrentes dos grandes inquéritos nacionais estão contribuindo no estudo das iniquidades sociais no consumo alimentar da população brasileira. Nos países em desenvolvimento, como é o caso do Brasil, a transição nutricional geralmente manifesta-se primeiro nos indivíduos de maior posição socioeconômica, como se pode perceber pelas altas prevalências de obesidade nessa população. Porém, com o avanço do desenvolvimento econômico e com a progressão da transição nutricional, esse padrão modifica-se e os grupos de menor posição socioeconômica passam a ter acesso a alimentos ricos em energia e pobres em nutrientes, tornando-se o principal grupo de risco para as $\mathrm{DCNT}^{40}$. Dessa forma, estudos com essa temática são essências para ajudar a compreender o complexo processo de transição nutricional brasileiro sob a perspectiva das desigualdades sociais.

A síntese de evidências desse estudo demonstrou que os homens têm um maior consumo de alimentos que fazem parte da dieta tradicional brasileira, além de consumirem mais frequentemente alimentos de risco para as DCNT. Já as mulheres têm um maior consumo de frutas e vegetais e sucos naturais, mas, ao mesmo tempo, tendem a trocar mais as refeições principais pelos lanches e sopas e a consumir mais doces. Além disso, os homens apresentam maiores percentuais de inadequação de micronutrientes do que as mulheres. Outros estudos demonstraram um consumo de padrões alimentares mais prudentes entre as mulheres brasileiras, principalmente naquelas acima dos 40 anos, com maior escolaridade e renda, enquanto os homens apresentam padrões alimentares mais tradicionais e de risco cardiovascular, também dependendo da renda e escolaridade ${ }^{41}$. Esses resultados chamam atenção para importância da investigação do papel de variáveis socioeconômicas nessa associação.

Nesse sentido, com análises que, para além do sexo, investiguem o papel da posição socioeconômica dos indivíduos, seria possível ter uma maior clareza das iniquidades no consumo alimentar entre homens e mulheres. É essencial se diferenciar sexo (biológico) de gênero (social). Sexo representa apenas a caracterização genética, anatômica e fisiológica do ser humano, já os papéis de gênero são construídos socialmente e são normalmente enquadrados como uma extensão de funções sociais biologicamente determinadas $^{41-43}$. Para que se possa analisar o gênero como um constructo de posição socioeconômica é preciso considerar os diferentes papéis sociais 


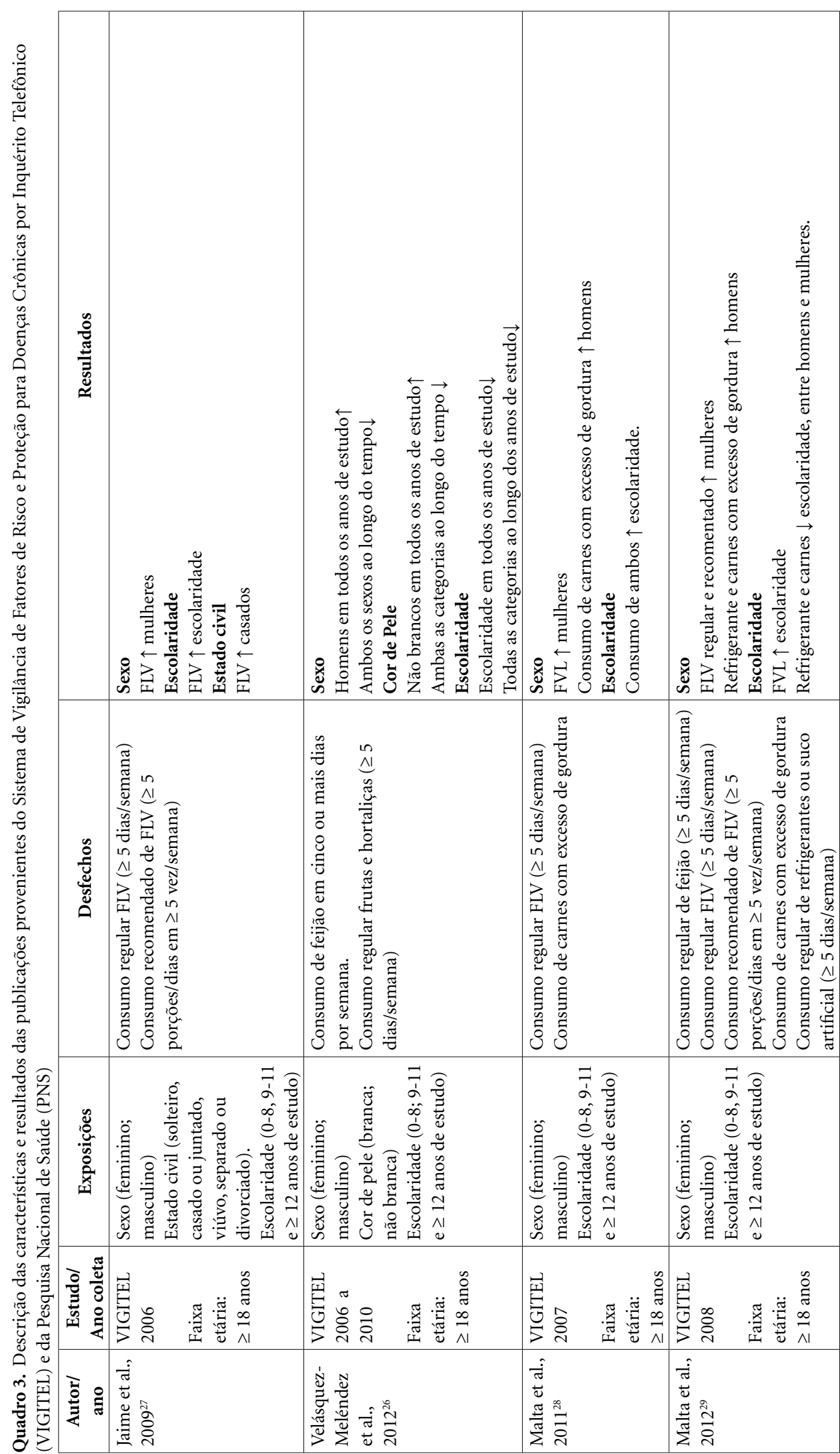




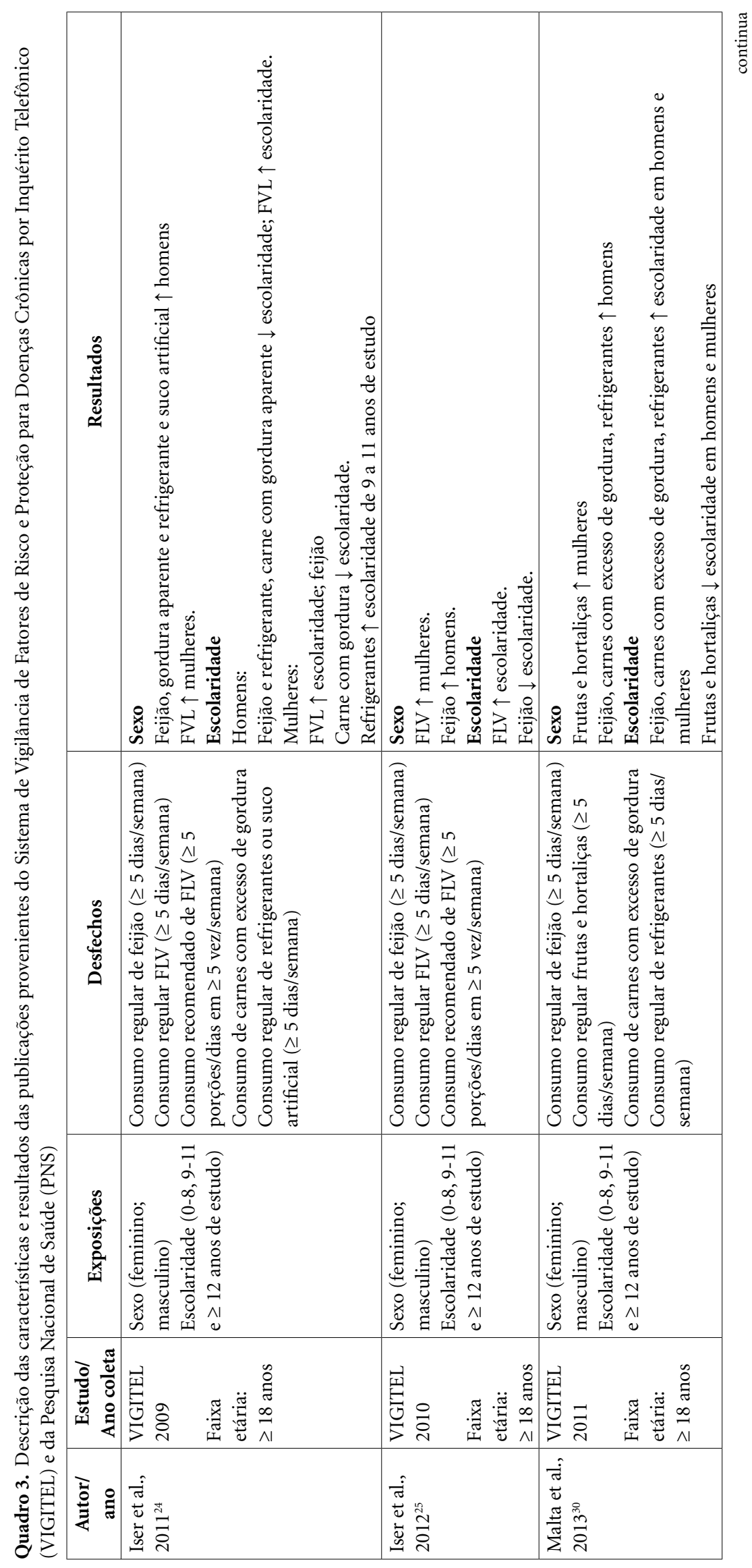




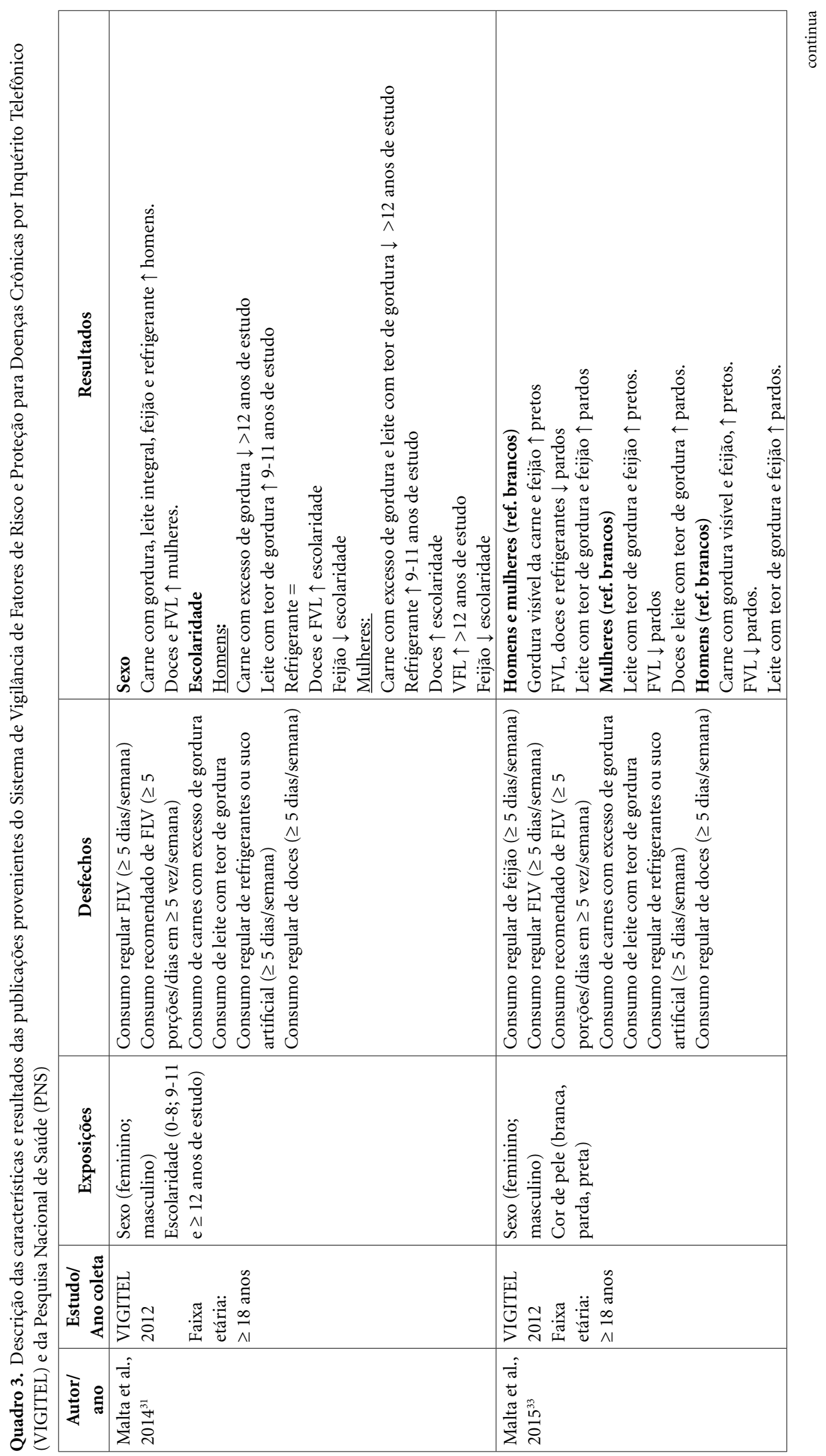




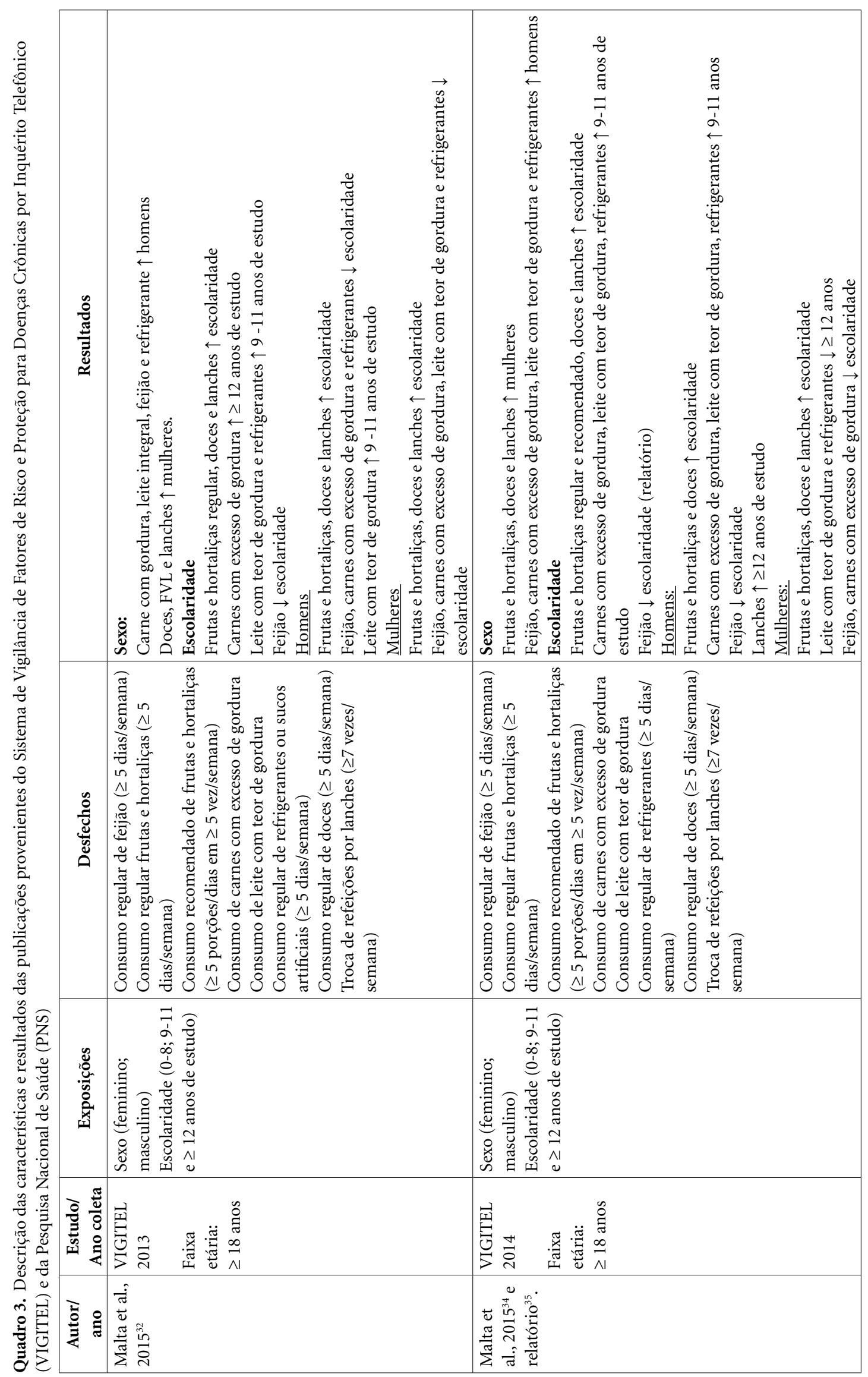




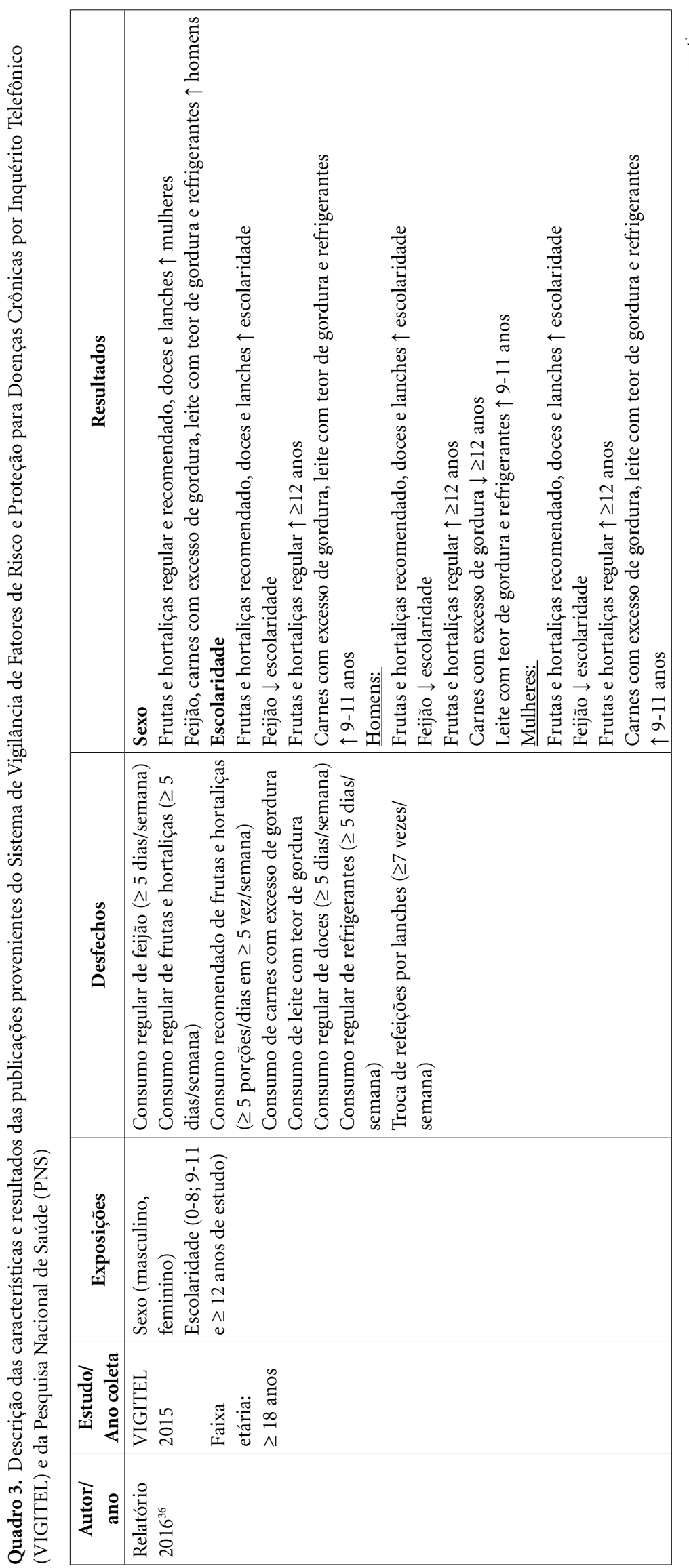




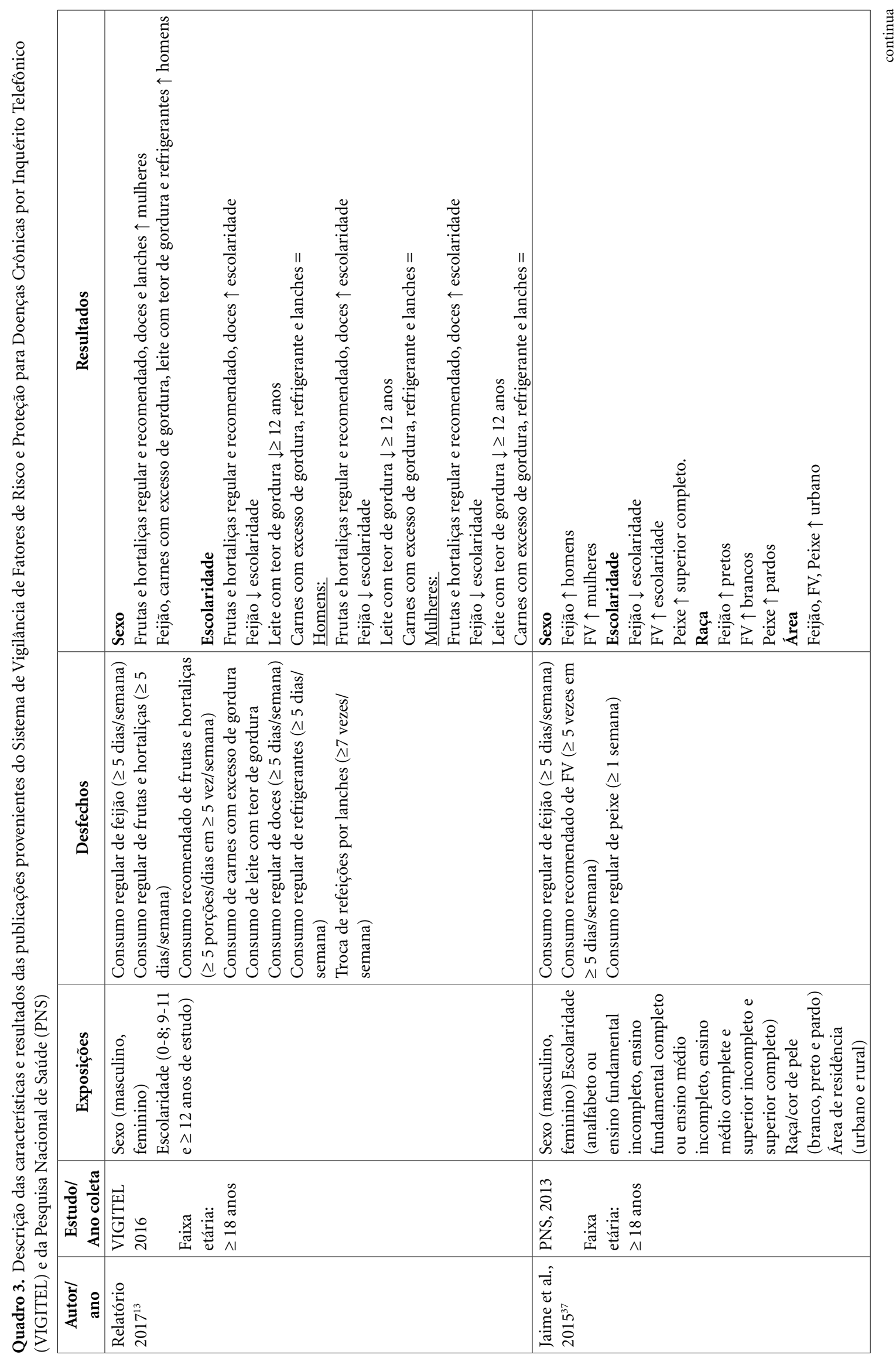




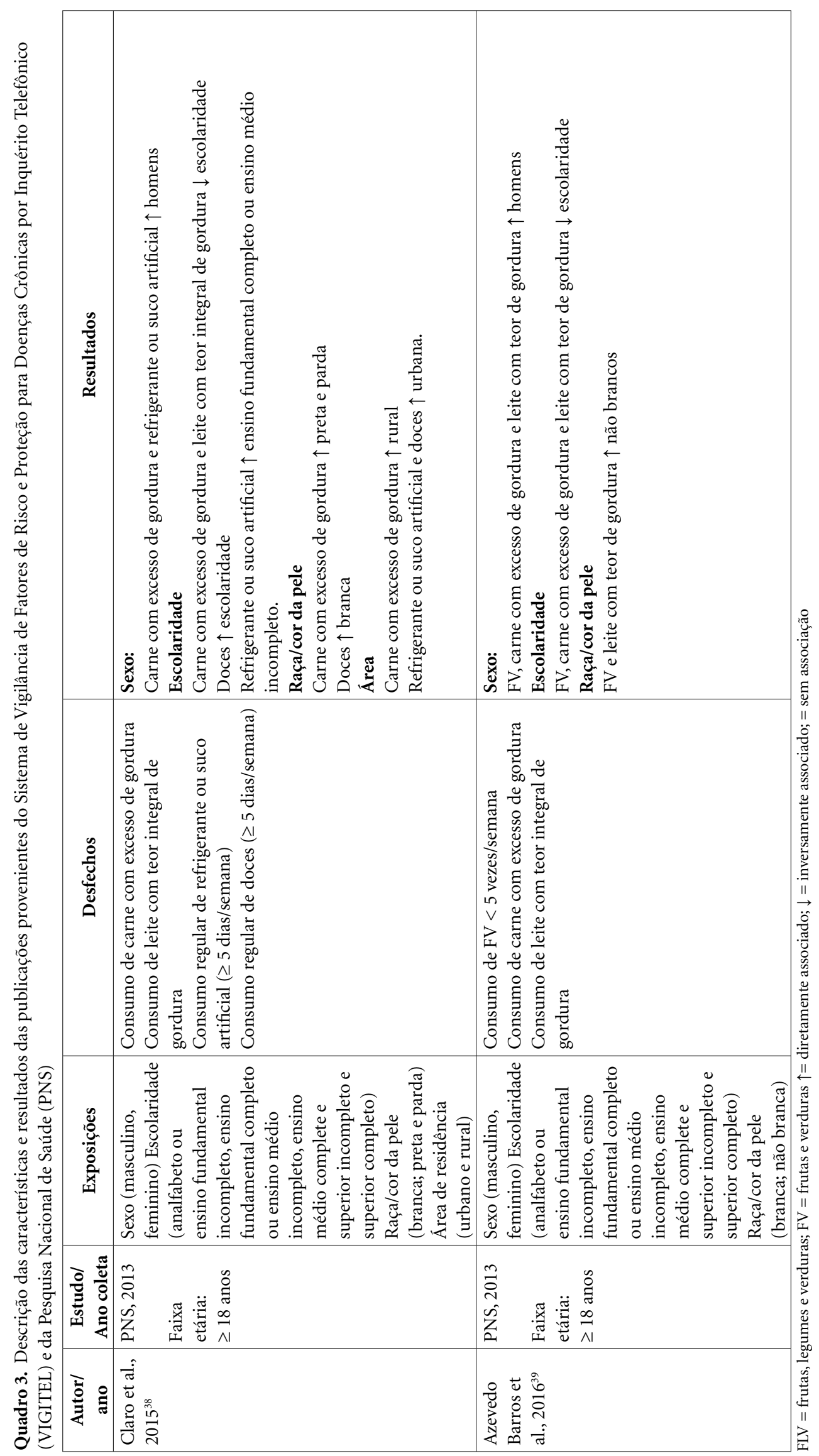


que homens e mulheres desempenham cotidianamente nos ambientes social, familiar, de trabalho e de lazer e as suas hierarquias ${ }^{8}$. Só assim será possível perceber que as iniquidades de gênero na saúde são em grande parte socialmente produzidas. Tais análises são especialmente importantes diante da necessidade de se compreender o desigual aumento da prevalência da obesidade entre as mulheres de baixa classe social no Brasil. Nessa revisão, apenas um estudo recuperado utilizou a nomenclatura gênero, porém não foram investigadas diferenças no consumo entre homens e mulheres, de acordo com outras variáveis de posição social, ou seja, segundo seus papeis sociais de gênero ${ }^{25}$.

Além disso, deve-se avançar na investigação da orientação sexual e outras identidades de gênero, uma vez que diversos estudos vêm apontando associações desiguais entre essas características e os hábitos alimentares, estado nutricional e $\mathrm{DCNT}^{44-47}$, possivelmente um efeito da conhecida discriminação e exclusão social vivida pela população de lésbicas, gays, bissexuais, travestis, transexuais e outras expressões de gênero ou sexualidade $(\mathrm{LGBT}+)^{48}$.

Pesquisadores têm chamado atenção para escassez de estudos empíricos que investiguem a magnitude e influência das desigualdades raciais na saúde no Brasil ${ }^{49}$. A influência da raça/ cor da pele no acesso/consumo de alimentos foi investigada por apenas três estudos. O principal marcador alimentar de proteção às DCNT, o consumo de frutas, legumes e verduras, foi maior entre os brancos. Por outro lado, os marcadores de risco estiveram associados à raça/cor da pele parda e/ou preta. O feijão, alimento tradicional da dieta do brasileiro, também esteve associado à raça/cor da pele parda e preta. Esses resultados indicam que a escolha dos alimentos pode estar associada ao seu valor monetário. Do ponto de vista cultural, a etnia exerce importante influência nos hábitos alimentares dos indivíduos. Já a raça/cor da pele, como categoria posição socioeconômica, em uma sociedade marcada pelo racismo, determina a trajetória de vida dos indivíduos com relação ao ambiente, ao acesso à educação, aos serviços de saúde, à ocupações e estilos de vida ${ }^{50}$. Nesse sentido, os estudos recuperados reduzem as medidas de cor da pele/raça para fins análiticos em branca e não branca ou branca, negra e parda.Tais medidas, em algumas situações, impedem a identificação adequada de populações negra e parda e, em maior proporção, de indivíduos que se autodeclaram amarelos e indígenas. Conhecer essas relações é fundamental, porque as disparidades raciais/étnicas na saúde têm sido tipicamente interpretadas como sinais de diferença genética/fisiológica ou, ao menos como diferenças culturais. Tais resultados sinalizam possíveis formas econômicas e sociais de discriminação racial ${ }^{8,44}$. Além disso, além dos inquéritos conduzidos entre populações tradicionais brasileiras, como indígenas e quilombolas, é de suma importância a investigação da influência da etnia no consumo alimentar nos grandes inquéritos nacionais para ajudar a compreender o impacto da transição nutricional na homogeneização da dieta dos brasileiros.

A situação conjugal é outra variável importante para o entendimento das dinâmicas de iniquidade em saúde. Nesta revisão, o único estudo que incluiu situação conjugal nas análises demonstrou que ser casado aumenta o consumo de frutas, legumes e verduras ${ }^{27}$. Internacionalmente, estudos têm associado a situação conjugal a diversos desfechos em saúde ${ }^{51-53}$, inclusive ao consumo alimentar, sugerindo que indivíduos casados teriam uma alimentação mais saudável do que os sem cônjuge ${ }^{6,54}$. O impacto da situção conjugal na saúde varia entre outros status, como: nunca ter casado, ser divorciado/separado ou ser viúvo ${ }^{53}$, demontrando a importância de investigações que incluam todas as categorias de análise. Além disso, análises dos determinantes sociais da saúde das mulheres apontam que essa associação têm interação com o gênero e outras condições socioeconômicas, como educação, trabalho e renda dos membros da famíliai ${ }^{54,55}$. Compreender essas interrelações é particularmente importante no Brasil, onde há um número crescente de mulheres de baixa renda que, sozinhas, são chefes de suas famílias e podem vivenciar importantes iniquidades no acesso/consumo dos alimentos. Além disso, para melhor compressão desse cenário, os estudos precisam avançar e incluir variáveis que possibilitem a compreensão das dinâmicas de exclusão social nas relações conjugais homoafetivas.

A renda e a escolaridade foram as principais variáveis utilizadas nos estudos para medir o nível socioeconômico dos indivíduos. Pelas características dos inquéritos, os produtos do Vigitel, da PNS e do INA apresentaram dados de escolaridade, já os produtos da POF utilizaram a renda familiar. Os indivíduos pertencentes às famílias com maior renda apresentaram um padrão alimentar contraditório, composto por uma alimentação mais diversificada e rica em nutrientes, que incluiu diversos alimentos in natura de maior valor monetário, mas também 
ao maior consumo de alimentos industrializados e refeições prontas. Por outro lado, a renda esteve inversamente associada ao consumo do padrão alimentar tradicional brasileiro, que é mais monótona e de menor valor monetário. Nesse sentido, uma importante iniquidade social parece persistir no Brasil, visto que indivíduos com maior renda têm maior possibilidade de escolha e, por outro lado, os indivíduos com menor renda parecem estar condicionados ao consumo de uma dieta básica e acessível. Esses resultados são semelhantes aos encontrados em recente revisão sistemática que investigou a associação entre nível socioeconômico e consumo alimentar em países em desenvolvimento ${ }^{40}$. Já nos países desenvolvidos, é mais clara a associação entre o maior nível socioeconômico e o consumo de uma alimentação saúdavel ${ }^{6,56}$.

A escolaridade esteve associada diretamente ao consumo de frutas, legumes e verduras e lanches (maior valor monetário) e inversamente ao consumo de feijão, peixe e farinha de mandioca (alimentos tradicionais). O padrão alimentar contraditório também esteve associado à escolaridade. Alimentos marcadores de uma alimentação de risco cardiovascular não apresentam um padrão de associação consistente com a escolaridade. Geralmente, considera-se que um maior nível de educação está associado a um maior nível de conhecimento em saúde e isso leva a um comportamento de saúde mais favorável ${ }^{57}$. Porém, os resultados dessa revisão são inconclusivos nesse sentido, sugerindo que a renda e a cultura estejam influenciando mais do que a escolaridade o consumo alimentar dos brasileiros.

Cabe também ressaltar que, apesar dos estudos que têm analisado as modificações no consumo alimentar dos brasileiros a partir do nível de processamento dos alimentos demonstrarem um maior consumo de alimentos in natura e minimante processados nos grupos de menor renda ${ }^{19}$, ao analisar a síntese de evidências neste estudo, percebe-se que o consumo de frutas, legumes, verduras e carnes, alimentos que contém nutrientes essenciais para saúde e que são habitualmente mais caros, ainda é maior entre os indivíduos de maior renda, reforçando a necessidade do continuo monitoramento do acesso a esses grupos de alimentos pela população.

Apenas as publicações oriundas das POF analisaram a área de moradia dos indivíduos, mesmo que a PNS também tenha representatividade para área urbanda e rural. $\mathrm{O}$ consumo de alimentos nas áreas rurais foi semelhante ao dos indivíduos de menor renda, ou seja, morar na área rural diminui a probabilidade dos indivíduos acessarem uma alimentação mais variada e saudável, inclusive de alimentos in natura. Pelas atividades agrícolas típicas da zona rural, imagina-se que os indivíduos residentes nessas áreas teriam maior acesso a alimentos in natura. Porém, indicadores socioeconômicos apontam uma grande desigualdade de renda, de escolaridade e nas condições de trabalho entre as áreas urbana e rural, o que pode, em parte, explicar os resultados encontrados. A renda na área urbana é 2,5 vezes maior a da área rural e o item alimentação representa a maior parte dos gastos das famílias na área rural ${ }^{58}$. Ao mesmo tempo, o distanciamento dos grandes centros de distribuição de alimentos explicaria a maior monotonia na dieta. Dessa forma, a área de moradia influencia tanto na sua posição socioeconômica quanto no acesso aos alimentos, sendo uma importante variável de vizinhança a ser melhor explorada nos estudos que avaliam as desigualdades no acesso/consumo alimentar entre os brasileiros.

Ao realizar essa revisão fica evidente que, para monitorar e compreender as desigualdades socioeconômicas, que podem levar à iniquidades na alimentação e nutrição dos brasileiros, é necessário não apenas a obtenção de dados sobre a saúde da população em relação às condições socioeconômicas. É preciso ter clareza conceitual sobre quais parâmetros socioeconômicos estão sendo medidos, como e porquê, além de sua relevância nas interpretações dos achados de pesquisa $^{59}$. É para esclarecer tais elementos que a teoria epidemiológica pode auxiliar: como guia do desenho de pesquisa até os métodos utilizados para a análise de dados. Se é possível perceber que variáveis não medidas pelos inquéritos e/ou não analisadas nas publicações poderiam auxiliar na compreensão das iniquidades sociais do binômio obesidade-desnutrição, também é necessário refletir sobre a teoria epidemiológica utilizada.

Nesse sentido, a teoria epidemiológica ecossocial coloca o grande desafio de conseguir explicações científicas sobre como estes diferentes fatores de discriminação social e econômica podem prejudicar a saúde das pessoas. A incorporação, em pesquisas, de indicadores tão distintos, que extrapolem as medidas socioeconômicas clássicas e meçam questões como experiências de discriminação, contextos histórico e biográfico, traumas sociais, exposição a ecossistemas degradados, entre outros, exige uma teoria epidemiológica capaz de medir tais fatores e explicar as implicações causais na saúde, no bem-estar e na saúde das pessoas em nível populacional ${ }^{59}$. A 
partir do marco teórico ora proposto, fica claro que a avaliação de iniquidades sociais e econômicas no acesso e no consumo de alimentos no Brasil têm uma importância fundamental para qualificar políticas públicas de saúde, alimentação e nutrição.

\section{Colaboradores}

\section{Referências}

R Canuto trabalhou na concepção, buscas e na redação final; M Fanton, na metodologia e discussão teórica; PIC Lira, na revisão e redação final do manuscrito.

1. Schmidt MI, Duncan BB, Silva GA, Menezes AM, Monteiro CA, Barreto SM, Chor D, Menezes PR. Chronic non-communicable diseases in Brazil: burden and current challenges. Lancet 2009; 377(9781):1949-1961.

2. Popkin BM, Adair LS, Ng SW. Global nutrition transition and the pandemic of obesity in developing countries. Nutr Rev 2012; 70(1):3-21.

3. Stringhini $S$, Carmeli C, Jokela $M$, Avendaño $M$, Muennig P, Guida F, Ricceri F, d'Errico A, Barros H, Bochud M, Chadeau-Hyam M, Clavel-Chapelon F, Costa G, Delpierre C, Fraga S, Goldberg M, Giles GG, Krogh V, Kelly-Irving M, Layte R, Lasserre AM, Marmot MG, Preisig M, Shipley MJ, Vollenweider P, Zins M, Kawachi I, Steptoe A, Mackenbach JP, Vineis $\mathrm{P}$, Kivimäki M. Socioeconomic status and the $25 \times 25$ risk factors as determinants of premature mortality: a multicohort study and meta-analysis of 1.7 million men and women. Lancet 2017; 389(10075):1229-1237.

4. Monteiro CA, Moura EC, Conde WL, Popkin BM. Socioeconomic status and obesity in adult populations of developing countries: a review. Bull World Health Organ 2004; 82(12):940-946.

5. Pampel FC, Denney JT, Krueger PM. Obesity, SES, and economic development: a test of the reversal hypothesis. Soc Sci Med 2012; 74(7):1073-1081.

6. Giskes K, Avendano M, Brug J, Kunst AE. A systematic review of studies on socioeconomic inequalities in dietary intakes associated with weight gain and overweight/obesity conducted among European adults. Obes Rev 2010; 11(6):413-29. 
7. Dinsa GD, Goryakin Y, Fumagalli E, Suhrcke M. Obesity and socioeconomic status in developing countries: a systematic review. Obes Rev 2012; 13(11):10671079.

8. Krieger N, Williams DR, Moss NE. Measuring social class in US public health research: concepts, methodologies, and guidelines. Annu Rev Public Health 1997; 18:341-378.

9. Allen L, Williams J, Townsend N, Mikkelsen B, Roberts N, Foster C, Wickramasinghe K. Socioeconomic status and non-communicable disease behavioural risk factors in low-income and lower-middle-income countries: a systematic review. Lancet Glob Health 2017; 5(3):277-289.

10. Comissão Nacional dos Determinantes Scociais em Saúde (CNDSS). As Causas Sociais das Iniquidades em Saúde no Brasil. Rio de Janeiro: Editora Fiocruz; 2008.

11. Instituto Brasileiro de Geografia e Estatística (IBGE). Aquisição Alimentar Domiciliar per Capita Brasil e Grandes Regiões. Rio de Janeiro: IBGE; 2004.

12. Instituto Brasileiro de Geografia e Estatística (IBGE). Pesquisa de orçamentos familiares 2008-2009: análise do consumo pessoal no Brasil. Rio de Janeiro: IBGE; 2011.

13. Brasil. Ministério da Saúde (MS). Vigitel Brasil 2016. Vigilância de fatores de risco e proteção para doenças crônicas por inquérito telefônico. Brasília: MS; 2017.

14. Instituto Brasileiro de Geografia e Estatística (IBGE). Pesquisa Nacional de Saúde: percepção do estado de saúde, estilos de vida e doenças crônicas: 2013. Rio de Janeiro: IBGE; 2014.

15. Enes CC, Silva MV. Disponibilidade de energia e nutrientes nos domicílios: o contraste entre as regiões Norte e Sul do Brasil. Cien Saude Colet 2009; 14(4):1267-1276.

16. Claro RM, Monteiro CA. Renda familiar, preço de alimentos e aquisição domiciliar de frutas e hortaliças no Brasil. Rev Saude Publica 2010; 44(6):1014-20.

17. Marchioni DM, Claro RM, Levy RB, Monteiro CA. Patterns of food acquisition in Brazilian households and associated factors: a population-based survey. $\mathrm{Pu}$ blic Health Nutr 2011; 14(9):1586-1592.

18. Levy RB, Claro RM, Mondini L, Sichieri R, Monteiro CA. Distribuição regional e socioeconômica da disponibilidade domiciliar de alimentos no Brasil em 20082009. Rev Saude Publica 2012; 46(1):6-15.

19. Martins APB, Levy RB, Claro RM, Moubarac JC, Monteiro CA. Participação crescente de produtos ultraprocessados na dieta brasileira (1987-2009). Rev Saude Publica 2013; 47(4):656-665.

20. Araujo MC, Verly Junior E, Junger WL, Sichieri R. Independent associations of income and education with nutrient intakes in Brazilian adults: 2008-2009 National Dietary Survey. Public Health Nutr 2014; 17(12):2740-2752.

21. Pereira RA, Souza AM, Duffey KJ, Sichieri R, Popkin BM. Beverage consumption in Brazil: results from the first National Dietary Survey. Public Health Nutr 2015; 18(7):1164-1172.

22. Araujo MC, Bezerra IN, Barbosa FDS, Junger WL, Yokoo EM, Pereira RA, Sichieri R. Consumo de macronutrientes e ingestão inadequada de micronutrientes em adultos. Rev Saude Publica 2013; 47(Supl. 1):177-189.
23. Souza ADM, Pereira RA, Yokoo EM, Levy RB, Sichieri R. Alimentos mais consumidos no Brasil: Inquérito Nacional de Alimentação 2008-2009. Rev Saude Publica 2013; 47(Supl. 1):190-199.

24. Iser BPM, Claro RM, Moura EC, Malta DC, Morais Neto OL. Fatores de risco e proteção para doenças crônicas não transmissíveis obtidos por inquérito telefônico - VIGITEL Brasil - 2009. Rev Bra Epidemiol 2011; 14(Supl. 1):90-102.

25. Iser BPM, Yokota RTC, Sá NNB, Moura L, Malta DC. Prevalência de fatores de risco e proteção para doenças crônicas nas capitais do Brasil - principais resultados do Vigitel 2010. Cien Saude Colet 2012; 17(9):2343-2356.

26. Velásquez-Meléndez G, Mendes LL, Pessoa MC, Sardinha LMV, Yokota RTC, Bernal RTI, Malta DC. Tendências da frequência do consumo de feijão por meio de inquérito telefônico nas capitais brasileiras, 2006 a 2009. Cien Saude Colet 2012; 17(12):3363-3370.

27. Jaime PC, Figueiredo ICR, Moura EC, Malta DC. Fatores associados ao consumo de frutas e hortaliças no Brasil, 2006. Rev Saude Publica 2009; 43(Supl. 2):5764.

28. Malta DC, Moura EC, Morais Neto OL. Desigualdades de sexo e escolaridade em fatores de risco e proteção para doenças crônicas em adultos Brasileiros, por meio de inquéritos telefônicos. Rev Bra Epidemiol 2011; 14(Supl. 1):125-135.

29. Malta DC, Silva SA, Oliveira PPV, Iser BPM, Bernal RTI, Sardinha LMV, Moura L. Resultados do monitoramento dos Fatores de risco e Proteção para Doenças Crônicas Não Transmissíveis nas capitais brasileiras por inquérito telefônico, 2008. Rev Bra Epidemiol 2012; 15(Supl. 1):639-650.

30. Malta DC, Iser BPM, Claro RM, Moura L, Bernal RTI, Nascimento AF, Silva Júnior JB, Monteiro CA Prevalência de fatores de risco e proteção para doenças crônicas não transmissíveis em adultos: estudo transversal, Brasil, 2011. Epidemiol Serv Saúde 2013; 22(4):423-434.

31. Malta DC, Bernal RTI, Nunes ML, Oliveira MM, Iser BPM, Andrade SSCA, Claro RM, Monteiro CA, Silva Júnior JB. Prevalência de fatores de risco e proteção para doenças crônicas não transmissíveis em adultos: estudo transversal, Brasil 2012. Epidemiol Serv Saude 2014; 23(4):609-622.

32. Malta DC, Campos MO, Oliveira MM, Iser BPM, Bernal RTI, Claro RM, Monteiro CA, Silva Júnior JB, Reis AAC. Prevalência de fatores de risco e proteção para doenças crônicas não transmissíveis em adultos residentes em capitais brasileiras, 2013. Epidemiol Serv Saude 2015; 24(3):373-387.

33. Malta DC, Moura L, Bernal RTI. Diferenciais dos fatores de risco de Doenças Crônicas não Transmissíveis na perspectiva de raça/cor. Cien Saude Colet 2015; 20(3):713-725

34. Malta DC, Stopa SR, Iser BPM, Bernal RTI, Claro RM, Nardi ACF, Reis AAC, Monteiro CA. Fatores de risco e proteção para doenças crônicas por inquérito telefônico nas capitais brasileiras, Vigitel 2014. Rev Bra Epidemiol 2015; 18(Supl. 2):238-255.

35. Brasil. Ministério da Saúde (MS). Vigitel Brasil 2014 Vigilância de fatores de risco e proteção para doenças crônicas por inquérito telefônico. Brasília: MS; 2015. 
36. Brasil. Ministério da Saúde (MS). Vigitel Brasil 2015. Vigilância de fatores de risco e proteção para doenças crônicas por inquérito telefônico. Brasília: MS; 2016.

37. Jaime PC, Stopa SR, Oliveira TP, Vieira ML, Szwarcwald CL, Malta DC. Prevalência e distribuição sociodemográfica de marcadores de alimentação saudável, Pesquisa Nacional de Saúde, Brasil 2013. Epidemiol Serv Saude 2015; 24(2):267-276.

38. Claro RM, Santos MAS, Oliveira TP, Pereira CA, Szwarcwald CL, Malta DC. Consumo de alimentos não saudáveis relacionados a doenças crônicas não transmissíveis no Brasil: Pesquisa Nacional de Saúde, 2013. Epidemiol Serv Saude 2015; 24(2):257-265.

39. Azevedo Barros MB, Lima MG, Medina LPB, Szwarcwald CL, Malta DC. Social inequalities in health behaviors among Brazilian adults: National Health Survey, 2013. Int J Equity Health 2016; 15:148.

40. Mayen AL, Marques-Vidal P, Paccaud F, Bovet P, Stringhini S. Socioeconomic determinants of dietary patterns in low- and middle-income countries: a systematic review. Am J Clin Nutr 2014; 100(6):1520-1531.

41. Canuto R, Camey S, Gigante DP, Menezes AMB, Olinto MTA. Análise de Componente Principal Focada: um método gráfico para explorar padrões alimentares. Cad Saude Publica 2010; 26(11):2149-2156

42. Annandale E, Hunt K. Gender Inequilites in Health. Buckingham: Open University Press; 2000.

43. Olinto MTA. Reflexões sobre o uso do conceito de gênero e/ou sexo na epidemiologia: um exemplo nos modelos hierarquizados de análise. Rev. Bras. Epidemiol 1998; 1(2):161-169.

44. Hatzenbuehler ML, McLaughlin KA, Slopen N. Sexual Orientation Disparities in Cardiovascular Biomarkers Among Young Adults. Am J Prev Med 2013; 44(6):612621.

45. Katz-Wise SL, Calzo JP, Scherer EA, Sarda V, Jackson B, Haines J, Austin SB. Sexual Minority Stressors, Internalizing Symptoms, and Unhealthy Eating Behaviors in Sexual Minority Youth. Ann Behav Med 2015; 49(6):839-852.

46. Rosario M, Corliss HL, Everett BG, Reisner SL, Austin SB, Buchting FO, Birkett M. Sexual Orientation Disparities in Cancer-Related Risk Behaviors of Tobacco, Alcohol, Sexual Behaviors, and Diet and Physical Activity: Pooled Youth Risk Behavior Surveys. Am J Public Health 2014; 104(2):245-254.

47. VanKim NA, Austin SB, Jun HJ, Hu FB, Corliss HL. Dietary Patterns during Adulthood among Lesbian, Bisexual, and Heterosexual Women in the Nurses' Health Study II. J Acad Nutr Diet 2017; 117(3):386-395.

48. Krieger N. Methods for the scientific study of discrimination and health: an ecosocial approach. $\mathrm{Am} \mathrm{J} \mathrm{Pu-}$ blic Health 2012; 102(5):936-944.
49. Chor D. Desigualdades em saúde no Brasil: é preciso ter raça. Cad Saude Publica 2013; 29(7):1272-1275.

50. Trostle JA. Epidemiologia e cultura. Rio de Janeiro: Editora Fiocruz; 2013.

51. Donoho CJ, Seeman TE, Sloan RP, Crimmins EM. Marital status, marital quality, and heart rate variability in the MIDUS cohort. J Fam Psychol 2015; 29(2):290-295

52. Han KT, Park EC, Kim JH, Kim SJ, Park S. Is marital status associated with quality of life? Health Qual Life Outcomes2014; 12:109.

53. Rendall MS, Weden MM, Favreault MM, Waldron H. The protective effect of marriage for survival: a review and update. Demography 2011; 48(2):481-506.

54. Haapala I, Prattala R, Patja K, Mannikko R, Hassinen M, Komulainen P, Rauramaa R. Age, marital status and changes in dietary habits in later life: a 21-year follow-up among Finnish women. Public Health Nutr 2012; 15(7):1174-1181.

55. Artazcoz L, Cortes I, Borrell C, Escriba-Aguir V, Cascant $\mathrm{L}$. Social inequalities in the association between partner/marital status and health among workers in Spain. Soc Sci Med 2011; 72(4):600-607.

56. Darmon N, Drewnowski A. Does social class predict diet quality? Am J Clin Nutr 2008; 87(5):1107-1117.

57. Dorner TE, Stronegger WJ, Hoffmann K, Stein KV, Niederkrotenthaler T. Socio-economic determinants of health behaviours across age groups: results of a cross-sectional survey. Central Eur J Med 2013; 125(910):261-269

58. Coelho AB, Aguiar DRD, Fernandes EA. Padrão de consumo de alimentos no Brasil. Rev Econ Sociol Ruralv 2009; 47(2):335-362.

59. Krieger N. Got Theory? On the 21st c. CE Rise of Explicit use of Epidemiologic Theories of Disease Distribution: A Review and Ecosocial Analysis. Curr Epidemiol Rep 2014; 1(1):45-56.

Artigo apresentado em 29/07/2017

Aprovado em 07/02/2018

Versão final apresentada em 09/02/2018 